\title{
Crystalline and amorphous structure of astrophysical ices
}

\author{
G. Strazzulla \\ Istituto Nazionale Astrofizica, Osservatorio Astrofisico di Catania, Catania, Italy \\ E-mail: gianni@oact.inaf.it
}

Received September 24, 2012

\begin{abstract}
The structure of water and other ices strongly depends on the temperature at which they formed, e.g., by vapor deposition. It is amorphous if ices are formed at low temperature (e.g., 10-30 K for water ice), or crystalline if the deposition temperature is higher $(140-150 \mathrm{~K})$. Ices have a "polycrystalline" structure at intermediate temperatures. The crystalline structure of ices can be damaged up to a complete amorphization by processes such as those due to energetic ion bombardment. Here I describe some experimental results obtained by ion irradiation of water and ammonia ices, two species particularly relevant in astrophysics. The results are discussed in the light of the relevance they have in astronomical environments where the actual structure of the ices depends on a competition between energetic processing that induce amorphization and thermal annealing that favors the transition towards more ordered structures.
\end{abstract}

PACS: $92.40 . \mathrm{Vv}$ Ice cores, ice sheets, ice shelves;

61.50.Ks Crystallographic aspects of phase transformations; pressure effects;

61.66.-f Structure of specific crystalline solids.

Keywords: astrophysical ice, crystalline structure, amorphous structure, ion irradiation.

\section{Introduction}

Earth based and space observations indicate that low temperature solids exist in many astrophysical environments. Condensed gases (ices) are present both in the interstellar medium as mantles on interstellar and circumstellar dust [1,2] and in/on many objects in the Solar System [3]. Ices are exposed to irradiation by energetic particles of different origins such as low energy cosmic rays, magnetospheric ions, solar flares and solar wind particles [4]. The structure (amorphous vs crystalline) of the astrophysical ices and other solids (silicates and carbons) depends on many parameters such as temperature and pressure at the time of their formation and the evolution during their long stay in space. There are several processes that drive structural changes, in particular those that induce crystalline order, as e.g. thermal annealing and those that favor damage and amorphization of the materials, as e.g. cosmic ion bombardment.

Here I present some results that have been obtained at the laboratory of experimental astrophysics (LASp) of INAF-Catania (Italy) by irradiating crystalline water or ammonia ices with energetic ions to study the induced damage and progressive (with ion flux) amorphization Some of the data have been previously published $[5,6]$ other data are presented here. Also described are the results obtained after irradiation of $\mathrm{H}_{2} \mathrm{O}: \mathrm{N}_{2}$ mixtures.

Water ice is, by far, the most abundant icy species in astrophysical environments [1,2]. Ammonia has been detect- ed in star forming regions [7] and it is very likely to be or to have been present at the time of the formation, on the solid surfaces in the Saturnian system (e.g. Clark et al. 1986). In particular, the bright moons (Mimas, Enceladus, Tethys, Dione, and Rhea) could be covered by magma ejected because of tidally driven heating [8]. Recently, the first firm identification of ammonia ice that has been found in the plumes emitted by Enceladus has been reported [9].

\section{Experimental apparatus}

Experiments have been performed at the Laboratorio di Astrofisica Sperimentale of INAF-Osservatorio Astrofisico di Catania (Italy) where a stainless steel high-vacuum, recently upgraded to ultra high-vacuum $\left(P<10^{-9}\right.$ mbar $)$ is used. The chamber is faced to a Danfysik $200 \mathrm{kV}$ ion implanter (for details see [10]). Targets are mounted at an angle of 45 degrees with respect to both the ion beam and the infrared beam of a FTIR spectrophotometer faced to the vacuum chamber. This allows to obtain "in-situ" spectra before and after each step of ion irradiation, without tilting or rotating the sample.

The thicknesses of the samples studied here are less than the penetration depth of the ions used $\left(200 \mathrm{keV} \mathrm{H}^{+}\right.$or $60 \mathrm{keV} \mathrm{Ar}^{++}$) that deposit a fraction of their energy in the ice and are stopped in the substrate (silicon or $\mathrm{KBr}$ ). The ion current density has been maintained low enough to avoid a macroscopic heating of the samples. The spectra were taken with a resolution of $1 \mathrm{~cm}^{-1}$. 
Water and or ammonia vapors were deposited at such a temperature (150 and $90 \mathrm{~K}$ respectively) that their structure is crystalline. The samples then have been cooled down to the chosen low $T(16-80 \mathrm{~K})$. At these temperatures they preserve crystalline structure and are irradiated to study the amophization process. We use the FTIR techniques because the band profiles are very sensitive to the structure of the species.

Mixtures $\mathrm{H}_{2} \mathrm{O}: \mathrm{N}_{2}=1: 1$, deposited at $20 \mathrm{~K}$, have an amorphous and porous structure. They have been irradiated to study the induced chemistry and the expected "compaction" of ice.

Fast ions penetrating solids deposit energy in the target by elastic and inelastic interactions that cause the breaking of molecular bonds. This produces physico-chemical modifications, erosion of material from the target and damage to the structure of the irradiated targets [4,10-12]. The energy released in the sample by impinging ions (dose) is given in $\mathrm{eV} / 16 \mathrm{u}$ since this is a convenient way to characterize chemical changes and compare the results obtained when irradiating different samples [13].

\section{Results}

In Fig. 1, the IR spectra of water ice in the range 8000 $4000 \mathrm{~cm}^{-1}(1.25-2.5 \mu \mathrm{m})$ at $80 \mathrm{~K}$ are shown. The three spectra, shifted for clarity, refer to (from top to bottom) amorphous ice, crystalline ice and irradiated $\left(200 \mathrm{keV} \mathrm{H}^{+}\right)$ crystalline ice at a dose of $3.2 \mathrm{eV} / 16 \mathrm{u}$. A few facts are clear from the figure: (a) both the peak position and the profile of the crystalline water ice bands are different from those of amorphous water ice; (b) particularly important is the appearance, in the crystalline ice, of a band centered at about $6050 \mathrm{~cm}^{-1}$ (1.65 $\left.\mu \mathrm{m}\right)$; (c) upon irradiation crystalline water ice becomes progressively amorphous.

The variation of the ratio of the area of the band at $6050 \mathrm{~cm}^{-1}(1.65 \mu \mathrm{m})$ to that of the band at $6660 \mathrm{~cm}^{-1}$

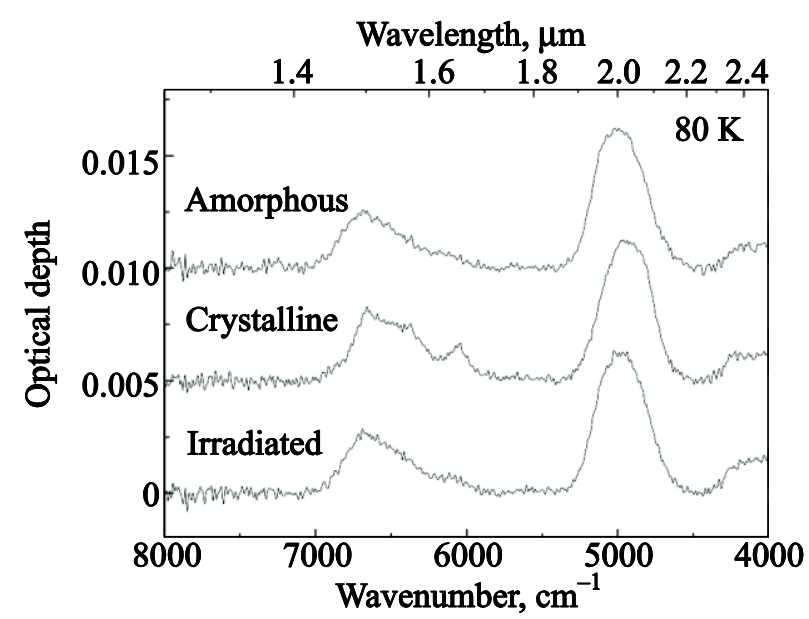

Fig. 1. IR spectra of water ice in the range $8000-4000 \mathrm{~cm}^{-1}$ $(1.25-2.5 \mu \mathrm{m})$ at $80 \mathrm{~K}$. The three spectra, shifted for clarity, refer to (from top to bottom) amorphous ice, crystalline ice and irradiated crystalline ice $\left(200 \mathrm{keV} \mathrm{H}^{+}\right)$at a dose of $30 \mathrm{eV} / 16 \mathrm{u}$.

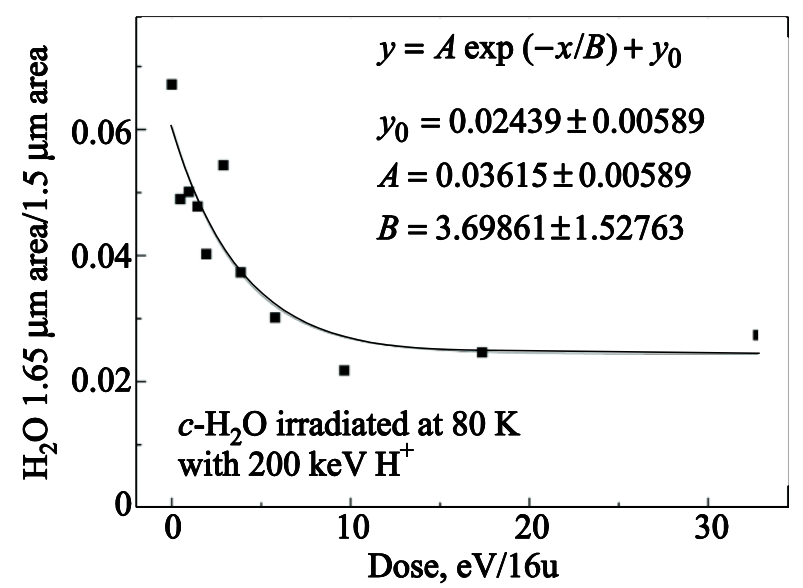

Fig. 2. The ratio of the area of the band at $6050 \mathrm{~cm}^{-1}(1.65 \mu \mathrm{m})$ to that of the band at $6660 \mathrm{~cm}^{-1}(1.5 \mu \mathrm{m})$ is plotted versus the dose released by $200 \mathrm{keV}$ protons irradiating a crystalline water ice film at $80 \mathrm{~K}$. The solid line is an exponential fit shown in the inset.

$(1.5 \mu \mathrm{m})$ is a good indicator of the effects induced by bombarding. In Fig. 2 this ratio is shown versus the dose released by $200 \mathrm{keV}$ protons irradiating a crystalline water ice film at $80 \mathrm{~K}$. Data points have been fitted by an exponential law as shown in the Figure. A progressive, with increasing dose, transition towards an amorphous phase is evident.

In Fig. 3, IR spectra of water ice in the range 3800$2800 \mathrm{~cm}^{-1}(2.63-3.57 \mu \mathrm{m})$ at $80 \mathrm{~K}$ are shown. The spectra refer to the as prepared crystalline ice, to the same sample irradiated $\left(200 \mathrm{keV} \mathrm{H}^{+}\right)$at different doses (up to of $3.2 \mathrm{eV} / 16 \mathrm{u})$. The spectrum of an amorphous sample of the same thickness is also shown for comparison.

Also for this band, due to the $\mathrm{O}-\mathrm{H}$ stretching modes, the peak position and the profile of the crystalline water ice

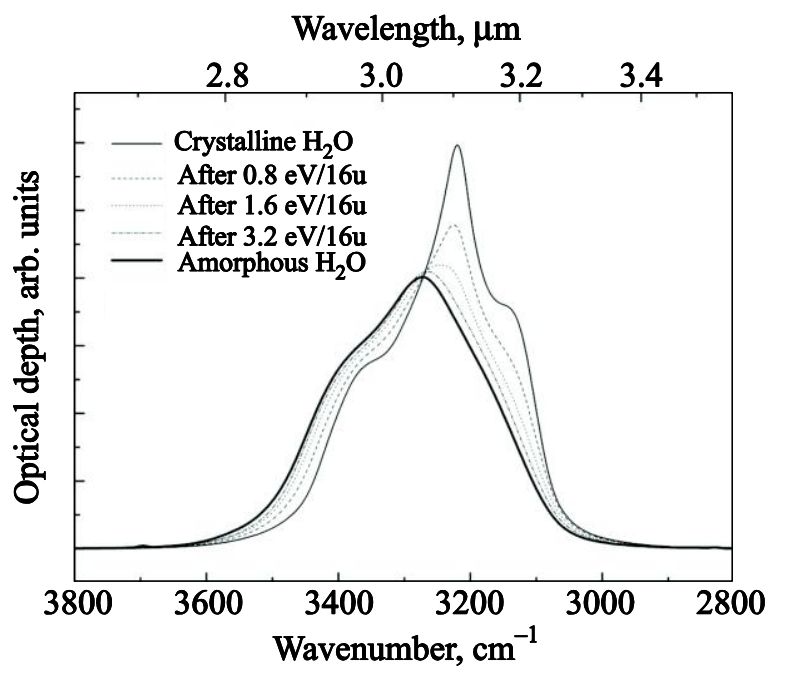

Fig. 3. IR spectra of water ice in the range $3800-2800 \mathrm{~cm}^{-1}$ $(2.63-3.57 \mu \mathrm{m})$ at $80 \mathrm{~K}$. The spectra refer to the as prepared crystalline ice, to the same sample irradiated $\left(200 \mathrm{keV} \mathrm{H}^{+}\right)$at different doses (up to of $3.2 \mathrm{eV} / 16 \mathrm{u}$ ). The spectrum of an amorphous sample of the same thickness is also shown for comparison. 


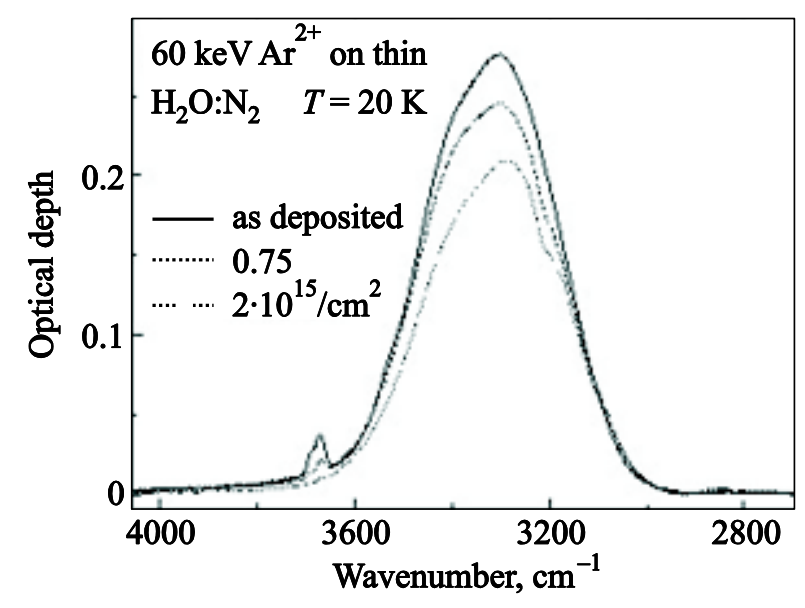

Fig. 4. IR spectra (in optical depth units) of a $\mathrm{H}_{2} \mathrm{O}: \mathrm{N}_{2}$ mixture as deposited at $20 \mathrm{~K}$ and after having been irradiated by $60 \mathrm{keV} \mathrm{Ar}^{2+}$.

bands progressively changes to become almost identical to that of amorphous ice, testifying for the ion induced structural change.

One question is to understand if the amorphous structure produced by irradiation of crystalline ice is identical to that of the amorphous state obtained after gas deposition at low temperature. The answer is no, they are not identical. As deposited amorphous ice is in fact porous as evidenced by the appearance in the spectrum of the $\mathrm{O}-\mathrm{H}$ dangling bond $(\mathrm{db})$ bands. These bands are attributed to three- and two-coordinate water molecules [14]. The peak positions of those features vary and for pure water ice is different from those for mixtures with other species (see Table 1 in [15]). Upon irradiation, ice is compacted and those features disappear $[15,16]$.

As a further proof of the induced compaction that testifies for the generality of the process, IR spectra (in optical depth units) of a $\mathrm{H}_{2} \mathrm{O}: \mathrm{N}_{2}$ mixture as deposited at $20 \mathrm{~K}$ and after having been irradiated by $60 \mathrm{keV} \mathrm{Ar}^{2+}$ are shown in Fig. 4. The spectral range is that corresponding to the stretching modes of frozen water that gives rise to the broad band. Also evident are bands at 3672 and $3694 \mathrm{~cm}^{-1}$ that as said, are due to the $\mathrm{O}-\mathrm{H}$ dangling bonds in porous, amorphous water ice. It is clear that in bombarded ice the $\mathrm{db}$ bands, which characterize frozen mixtures water/nitrogen, disappear due to the ice compaction.

The IR spectra of ammonia ice $(80 \mathrm{~K})$ in the range $3600-1400 \mathrm{~cm}^{-1}(2.78-7.14 \mu \mathrm{m})$ are shown in Fig. 5. The spectra refer to the as prepared crystalline ice, to the same sample irradiated with $60 \mathrm{keV} \mathrm{Ar}{ }^{2+}$ ata dose of $33 \mathrm{eV} / 16 \mathrm{u}$. The spectrum of an amorphous sample is also shown for comparison. In this case the conversion of the original crystalline structure to an amorphous one is evident.

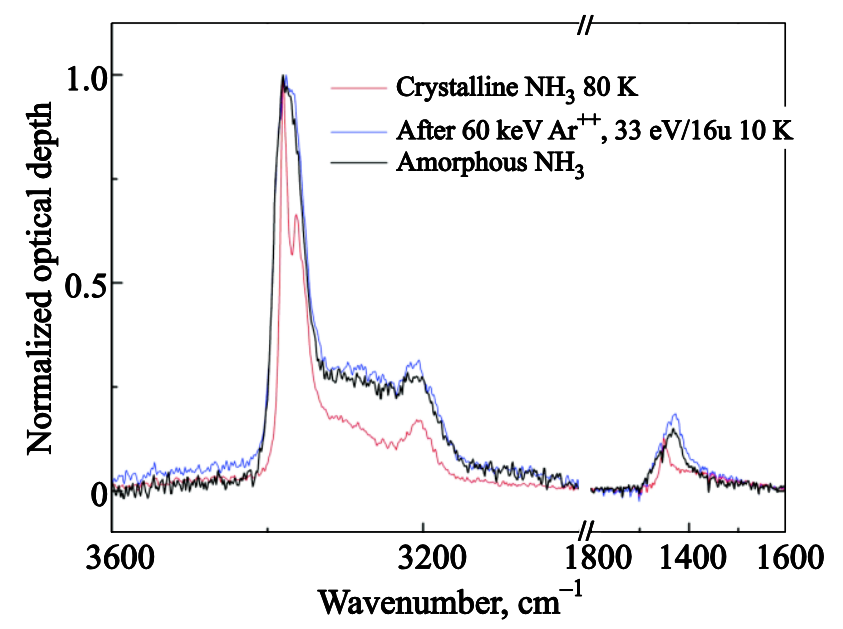

Fig. 5. IR spectra of ammonia ice in the range $3600-1400 \mathrm{~cm}^{-1}$ $(2.78-7.14 \mu \mathrm{m})$ at $80 \mathrm{~K}$. The spectra refer to the as prepared crystalline ice and to the same sample irradiated $\left(60 \mathrm{keV} \mathrm{Ar}^{2+}\right)$ at a dose of $33 \mathrm{eV} / 16 \mathrm{u}$. The spectrum of an amorphous sample is also shown for comparison.

\section{Discussion}

IR spectroscopy is a powerful technique to investigate the structure of the ices as shown above for water and ammonia ice. It is used in astrophysics to investigate the structure of ices in astrophysical environments and their evolution. The structure of the ice depends in fact on the temperature and pressure at the time of their formation, on the subsequent thermal evolution and, as discussed above, on the amount of processing (ion bombardment, but also photoprocessing, see e.g. [17]) they are exposed to.

Ice mantles of grains in dense clouds in the interstellar medium are amorphous and compact. Most likely, this reflect the fact that they are formed at low temperature by surface reaction of $\mathrm{O}$ (and/or $\mathrm{O}_{2}$ and/or $\mathrm{O}_{3}$ ) and $\mathrm{H}$ (or $\mathrm{OH}$ ) on the cold refractory dust as experimentally evidenced [18-21]. Particularly relevant is the recent finding that molecular hydrogen reacting with $\mathrm{OH}$ can also produce compact amorphous ice [22].

The structure of the ice can change when clouds collapse to form young stars, which heat up their circumstellar envelopes. Under such circumstances a transition to a crystalline structure (or to evaporation when the temperature increases further) is expected [23]. At the same time, particle and photon fluxes increase and this also also favors amorphization of the crystalline ices. Such a scenario has been recently observationally evidenced [24]. These authors observed a $T$ Tauri star (YLW 16 A in the $\rho$ Ophiuchi molecular cloud) and showed that crystallinity increases in the upper layers of the circumstellar disk, while only amorphous grains exist in the bipolar envelope. This implies that water ice crystallizes and remains crystallized close to the disk atmosphere where it is shielded against hard irradiation. 
The competition between crystallization and amorphization also occurs on some objects in the Solar System (see e.g. [3]). In fact, ices on the surfaces of the moons in the external solar system exhibit a variety of structures [25]. The three major Jovian icy moons exhibit different structures: ice is mostly amorphous on the superficial layers of Europa (where the temperature is lower and ion fluxes are high) and crystalline on Callisto (that has the highest temperature), while both structures are found on Ganymede [26]. Roughly, the distribution of crystalline and amorphous surface ice on Ganymede is consistent with the expected distribution of energetic magnetospheric ions and neutrals, although there are not yet enough spatially resolved data to confirm the relation between the local fluxes of energetic particles and the structure of the ice.

In conclusion, I believe that laboratory data like those I discussed here are of the great relevance for understanding how astronomical observations can be used to reconstruct the evolution of ices in many astronomical environments.

\section{Acknowledgements}

This research has been partially supported by the European COST Action CM 0805: The chemical cosmos: Understanding Chemistry in Astronomical Environments. The experimental work in Catania has been performed in the last years by the Laboratorio di Astrofisica SPerimentale (LASP) team and co-workers (G.A. Baratta, R. Brunetto, R. DiBenedetto, D. Fulvio, M. Garozzo, V. Greco, S. Ioppolo, F. Islam, Z. Kanuchova, G. Leto, M.E. Palumbo, I. Sangiorgio, F. Spinella, G. Strazzulla et al.).

1. E.L. Gibb, D.C.B. Whittet, A.C.A. Boogert, and A.G.G.M. Tielens, ApJ 151, 35 (2004).

2. L.A. Allamandola, M.P.Bernstein, S.A. Sandford, and R.L. Walker, SSRev. 90, 219 (1999).

3. J.B. Dalton,D .P. Cruikshank, K. Stephan, T.B. McCord, A. Coustenis, R.W. Carlson, and A. Coradini, SSRev. 153, 113 (2010).

4. G. Strazzulla, NIMB 269, 842 (2011).

5. G.A. Baratta, F. Spinella, G. Leto, G. Strazzulla, and G. Foti, A\&A 252, 421 (1991).

6. G. Leto, O. Gomis, and G. Strazzulla, Mem. SAIS 6, 57 (2005).
7. K.I. Öberg, A.C.A. Boogert, K.M. Pontoppidan, S. van den Broek, E.W. van Dishoeck, S. Bottinelli, G.A. Blake, and N.J.II Evans, ApJ 740, id. 109 (2011).

8. K. Multhaup and T. Spohn, Icarus 186, 420 (2007).

9. J.H. JrWaite, W.S. Lewis, B.A. Magee, J.H. Waite, Jr., W.S. Lewis, B.A. Magee, J.I. Lunine, W.B. McKinnon, C.R. Glein, O. Mousis, D.T. Young, T. Brockwell, J. Westlake, M.-J. Nguyen, B.D. Teolis, H.B. Niemann, R.L. McNutt, Jr., M. Perry, and W.-H. Ip, Nature 460, 487 (2009).

10. G. Strazzulla, G.A. Baratta, and M.E. Palumbo, Spectrochim. Acta A 57, 825 (2001).

11. R.A. Baragiola, R.A. Vidal, W. Svendsen, J. Schou, M. Shi, D.A. Bahr, and C.L. Atteberry, NIMB 209, 294 (2003).

12. R.E. Johnson, R.w. Carlson, J.F. Cooper, C. Paranicas, M.H. Moore, and M.C. Wong, in: Jupiter: Planet, Satellites, and Magnetosphere, F. Bagenal, W. McKinnon, and T. Dowling (eds.), Cambridge Univ. Press, UK, 485 (2004), p. 485.

13. G. Strazzulla and R.E. Johnson, in: Comets in the post-Halley Era, R.L. Newburn, Jr., M. Neugebauer, and J. Rahe (eds.), Kluwer, Publ. Co., London, 243 (1991), p. 243.

14. B. Rowland, M. Fisher, and J.P. Devlin, J Chem. Phys. 95, 1378 (1991).

15. M.E. Palumbo, $A \& A$ 453, 903 (2006).

16. U. Raut, M. Famá, M.J. Loeffler, and R.A. Baragiola, ApJ 687, 1070 (2008).

17. G. Leto and G.A. Baratta, $A \& A$ 397, 7 (2003).

18. S. Ioppolo, H.M. Cuppen, C. Romanzin, E.F. van Dishoeck, and H. Linnartz, ApJ 686, 1474 (2008).

19. Y. Oba, N. Miyauchi, H. Hidaka, T. Chigai, N. Watanabe, and A. Kouchi, ApJ 701, 464 (2009).

20. H. Mokrane, H. Chaabouni, M. Accolla, E. Congiu, F. Dulieu, M. Chehrouri, and J.L. Lemaire, ApJ 705, L195 (2009).

21. F. Dulieu, L. Amiaud, E. Congiu, J.-H. Fillion, E. Matar, A. Momeni, V. Pirronello, and J.L. Lemaire, $A \& A$ 512, id. A30 (2010).

22. Y. Oba, N. Watanabe, T. Hama, K. Kuwahata, H. Hidaka, and A. Kouchi, ApJ 749, id. 67 (2012).

23. E. Dartois and L. D'Hendecourt, $A \& A$ 365, 144 (2001).

24. A.A. Schegerer and S. Wolf, $A \& A$ 517, A87 (2010).

25. W.M. Grundy, M.W. Buie, J.A. Stansberry, J.R. Spencer, and B. Schmitt, Icarus 142, 536 (1999).

26. G.B. Hansen and T.B. McCord, Proc. J. Geophys. Res. 109, E01012 (2004). 